\title{
A Collaborative Healthcare Service Framework and Performance Evaluation
}

\author{
John Jung-Woon Yoo', Krishnaveni Gnanasekaran'2, Chen-Yang Cheng 3 \\ ${ }^{1}$ Department of Industrial \& Manufacturing Engineering \& Technology, Bradley University, Peoria, IL, USA \\ ${ }^{2}$ Information Analytics, Caterpillar Inc., Peoria, IL, USA \\ ${ }^{3}$ Department of Industrial Engineering and Enterprise Information, Tunghai University, Taichung, Taiwan \\ Email: jyoo@bradley.edu, gnanasekaran krishnaveni@cat.com,chengcy@thu.edu.tw
}

Received 12 April 2014; revised 12 May 2014; accepted 20 May 2014

Copyright (C) 2014 by authors and Scientific Research Publishing Inc.

This work is licensed under the Creative Commons Attribution International License (CC BY).

http://creativecommons.org/licenses/by/4.0/

(c) (i) Open Access

\begin{abstract}
While collaboration is becoming increasingly critical in many industries to maximize revenue and minimize cost by taking advantage of other's expertise, healthcare industry has hardly utilized the advantages of collaboration. This paper proposes a framework for collaborative healthcare services. The proposed framework is based on Service Oriented Architecture using web services and utilizes a medical coding system, Current Procedure Terminology (CPT), for interoperability. The framework is designed to facilitate collaborations among healthcare service providers, and is expected to reduce medical expenditure of patients and increase revenue of healthcare service providers. In order to verify the performance of the proposed framework, a simulation study is conducted. The experimental results show that the framework contributes to utilization increase and treatment lead time decrease in healthcare services.
\end{abstract}

\section{Keywords}

Collaboration, Medical Coding System, Service Oriented Architecture, Simulation

\section{Introduction}

Collaboration is a key success factor in many businesses. Manufacturers design a product together with many suppliers and outsource parts to make the product. For example, Intel, Samsung, LG and Sharp are major suppliers of parts for Apple's flagship product-iPhone, and they collaborate even from the initial stage of a product design. The goal of collaboration is to maximize product performance and minimize costs by taking advantage of specialties of collaborating partners. Boeing's strategy for developing the 787 Dreamliner is another good example. Boeing has partnered with fifteen companies in ten U.S. states and seven countries to create the 
major structural systems of the aircraft [1].

While collaboration has been utilized in many industries and contributed to cost reduction and performance improvement, healthcare industry has rarely utilized the advantages of collaboration. Very few literatures have addressed the collaboration issues among healthcare service providers [2]-[4] and recently medical knowledge collaboration in cloud computing has been investigated [5]. Collaboration in healthcare services is defined as collective activities among healthcare service providers to provide high quality services for service users and to increase revenue. The limited studies consider pricing behaviors or implications of medical outsourcing. In this paper, we propose a collaborative healthcare service framework to provide better services for patients and at the same time to increase revenues of collaborating hospitals. For example, if a patient exhibiting symptoms of liver cancer is recommended by a hospital specialist to undergo a series of diagnostic tests that might take several months to administer due to the hospital's limited staff or equipment availability, such a lengthy period of diagnosis may not be appropriate for treating the patient, or other patients with acute illnesses that require more immediate medical attention. If the hospital can outsource some of the required tests to other hospitals that have the available resources to perform the tests more quickly, not only would the patient benefit from more expeditious service, it might even encourage price competition among these hospitals, resulting in cost savings for the outsourcing hospital as well as the patient. This liver cancer diagnosis example illustrates the advantages of healthcare service collaboration for both patients and hospitals.

Increased utilization of resources in hospitals can also help reduce medical costs. Table 1 shows itemized Magnetic Resonance Imaging (MRI) cost. Equipment purchasing, maintenance and operating personnel salary comprise major fixed cost to operate MRI equipment. As more tests are conducted on MRI equipment, the overhead cost of operating the machine can be shared by the tests being done on the MRI equipment. According to Table 1 [4], in case of one thousand tests performed on one machine, the cost per test can be regarded as $\$ 790$; in case of two thousand tests, the cost can be reduced to $\$ 441$. In summary, if the utilization of the equipment increases, the testing cost can be reduced. This MRI cost analysis clearly shows the need for the proposed collaboration framework to increase the utilization of medical resources.

In this paper, an information system framework that supports healthcare service collaboration among healthcare service providers is proposed and a simulation study is conducted to test the viability of the proposed framework. This paper is organized as follows: Section 2 reviews previous literature and justifies the contribution of this paper. Section 3 presents the information system architecture and key technologies for the proposed framework for healthcare service collaboration. In the section, possible liver cancer diagnosis scenarios based on the proposed framework are introduced. Section 4 discusses the result of a simulation study that supports the advantages of the proposed framework. Finally, Section 5 concludes the paper with the contribution and limitation of the proposed ideas as well as future research topics.

\begin{tabular}{ccccc}
\multicolumn{2}{l}{ Table 1. Itemized MRI cost. } \\
$\begin{array}{c}\text { Expense } \\
\text { Category }\end{array}$ & $\begin{array}{c}\text { Type of } \\
\text { Expense }\end{array}$ & $\begin{array}{c}\text { Operational } \\
\text { Cost/Year }\end{array}$ & $\begin{array}{c}\text { Cost per } \\
1000 \text { Exams }\end{array}$ & $\begin{array}{c}\text { Cost per } \\
2000 \text { Exams }\end{array}$ \\
\hline MRI Equipment & Fixed & $\$ 300,000$ & $\$ 300$ & $\$ 150$ \\
Maintenance & Fixed & $\$ 100,000$ & $\$ 100$ & $\$ 50$ \\
Space & Fixed & $\$ 50,000$ & $\$ 50$ & $\$ 25$ \\
Personnel & Fixed & $\$ 125,000$ & $\$ 125$ & $\$ 63$ \\
Overhead & Fixed & $\$ 50,000$ & $\$ 50$ & $\$ 25$ \\
Office Expenses & Fixed & $\$ 15,000$ & $\$ 15$ & $\$ 8$ \\
Marketing & Fixed & $\$ 10,000$ & $\$ 10$ & $\$ 5$ \\
Utilities & Fixed & $\$ 50,000$ & $\$ 50$ & $\$ 25$ \\
Medical Supplies & Variable & $\$ 25$ & $\$ 25$ & $\$ 25$ \\
Film & Variable & $\$ 30$ & $\$ 30$ & $\$ 30$ \\
Billing \& Collect & Variable & $\$ 35$ & $\$ 35$ & $\$ 35$ \\
Total & & & $\$ 790$ & $\$ 441$ \\
\hline
\end{tabular}




\section{Literature Review}

Collaboration in healthcare service areas, particularly at the hospital level, has not been common. Medical collaboration not only means sending medical records electronically from one hospital to another hospital, but it also means collective activities among healthcare service providers for diagnosis or treatment processes for a patient. Collaboration among hospitals can facilitate access to quality care for patients [3]. Furthermore, we claim in this paper that collaboration among hospitals can increase the utilization of medical resources at hospitals and ultimately reduce healthcare service cost for patients.

The utilization of expensive medical resources has been a major issue for hospitals. It is not uncommon for multiple competing MRI (Magnetic Resonance Imaging) centers to go half utilized and even far less than that [6]. Planning and scheduling approaches can be used to improve operation room utilization within a hospital if the utilization of medical resources of a hospital fluctuates significantly [7]. However, there are cases in which medical resources of a hospital are in near or above $100 \%$ utilization, while those of another hospital are severely underutilized. Collaboration among hospitals using information systems can solve such problems by providing information on the availability of the hospitals, so that patients can get timely healthcare services from hospitals that have the needed resources available.

Although collaborative healthcare services among healthcare service providers have not been common, the infrastructure for medical collaboration has been developed and implemented among medical organizations. Most hospitals utilize inter- or intra-hospital information systems (HIS), managing information on patients, appointment status, schedule of doctors and nurses, and billing. Furthermore, electronic health records (EHR) systems [8] have been implemented in order to exchange patient and examination information among healthcare service providers. Medical communication standards play a key role in information exchange, such as Health Level Seven (HL7) [9] and Digital Imaging and Communications in Medicine (DICOM) [10]. HL7 protocol is designed for text-based data exchange and is commonly used among 95 percent of U.S. hospitals [11], while DICOM is designed for image-based data exchange.

Web services have been applied to support inter-hospital communications and can facilitate collaboration among healthcare service providers and improve the quality of healthcare services [12]. Some of the key benefits of web services technology are platform independence, highly flexible collaboration capability among heterogeneous systems, and resource sharing. A modeling process for medical web services implemented two sample transactions, patient registration and image retrieval, using Biztalk 2004 [13]. Furthermore, the automation of medical web service composition considering semantic issues using ontology has been investigated [14]. They have proposed a framework that describes how to represent the compositional knowledge and how to interoperate heterogeneous healthcare services, and built an ontology integration method, called SEMIO, which stands for Semantic Interoperability.

Medical coding systems have significantly contributed to the interoperability between healthcare service providers and insurance companies. Healthcare service collaboration cannot work without interoperation among heterogeneous systems that each hospital utilizes. One of the widely used medical coding systems is CPT (Current Procedural Terminology) codes [15]. A CPT code is a five-digit alphanumeric identifier of healthcare services. In this context, healthcare services include any activities performed by healthcare service providers, such as medical treatments, surgical operations or diagnostic examinations. For example, a CPT code of 90663 denotes influenza virus vaccination for $\mathrm{H} 1 \mathrm{~N} 1$ and 76815 denotes pregnant uterus ultrasound. The CPT coding system has been used mostly for billing purposes. When hospitals send billing documents to insurance companies, they use the CPT code to indicate which healthcare services are provided. In addition, a CPT code can be used to check the coverage of the insurance policy of a patient.

The CPT coding system has contributed to ensuring uniformity among medical organizations. The same CPT code used in two hospitals means the same healthcare service, such as appendectomy or endoscopy, but does not mean the same healthcare service fee. One hospital may charge a higher fee than the other hospital for the same CPT code, which means the coding system is for the identification of healthcare services, not for the decision of service fees. On the other hand, Healthcare Common Procedure Coding System (HCPCS) [15] provides not only the identification code but also the service fee information. The HCPCS coding system is designed by the Centers for Medicaid \& Medicare Services in the United States for uniformity in both healthcare service codes and billing procedures for its Social Security program, which is a medical insurance program for seniors and people with disability. HCPCS uses the same CPT codes, but assigns a fixed fee to each code.

As discussed in this section, medical collaboration has not been common in current healthcare services, but 
underlying technologies for the collaboration has been established. Medical collaboration is expected to contribute to timely services with reduced cost for patients and revenue increase for hospitals by increased utilization. This paper proposes a framework for collaborative healthcare services. The proposed framework is based on Service Oriented Architecture using web services and utilizes the CPT medical coding system.

\section{Collaborative Healthcare Framework}

\subsection{System Architecture}

The proposed framework for collaborative healthcare services is based on Service Oriented Architecture (SOA) [16], which is underlying architecture for web services technology. Web services technology is the most widely accepted technology to integrate services provided by service providers on the Internet. One of the key features of web services technology is that it provides interoperability for service providers and users. Interoperability means an ability that multiple systems can communicate with one another no matter which operating systems or software programs individual systems are based on.

The conceptual diagram for the proposed collaborative healthcare service framework is illustrated in Figure 1, which consists of three main players, i.e., a medical collaboration broker, healthcare service users, and healthcare service providers who participate in collaborative services. The collaboration broker maintains up-to-date service-related information of hospitals that participate in collaboration. The information includes a list of healthcare services provided by each healthcare service provider, detailed input and output data from the provided healthcare services, hospital contact information, among others. The healthcare service broker system identifies most suitable health care services provided by one of the collaborating hospitals according to patients' search requests for a particular healthcare service. The healthcare service identification process by the service broker system can be extended to create complete healthcare service workflows. Taking the aforementioned example of the liver cancer diagnosis case, if the patient needs to get abdominal ultrasound, CT scan, MRI, and liver biopsy for diagnosis, the four tests have sequence requirements established by the primary doctor of a patient, in which the test order must be followed. Abdominal ultrasound is set to be performed first. After the ultrasound test, there are no sequences requirements between MRI and CT scan, which means either scan can start before the other. However, both should be completed before liver biopsy. These sequence requirements of the four tests will result in a workflow. The collaboration broker generates a list of tests satisfying the sequence requirements specified in the workflow. When the broker generates the list of tests, it should consider the patient's goal. If the goal of the patient is to receive all services within a minimal amount of time, then the patient needs the earliest completion schedule for the whole series of tests. On the other hand, if the goal of the patient is to receive all services at a minimal cost, then the patient needs the most inexpensive way to receive the whole set of services.

In order for the medical collaboration broker to identify most-suitable healthcare service or a series of health
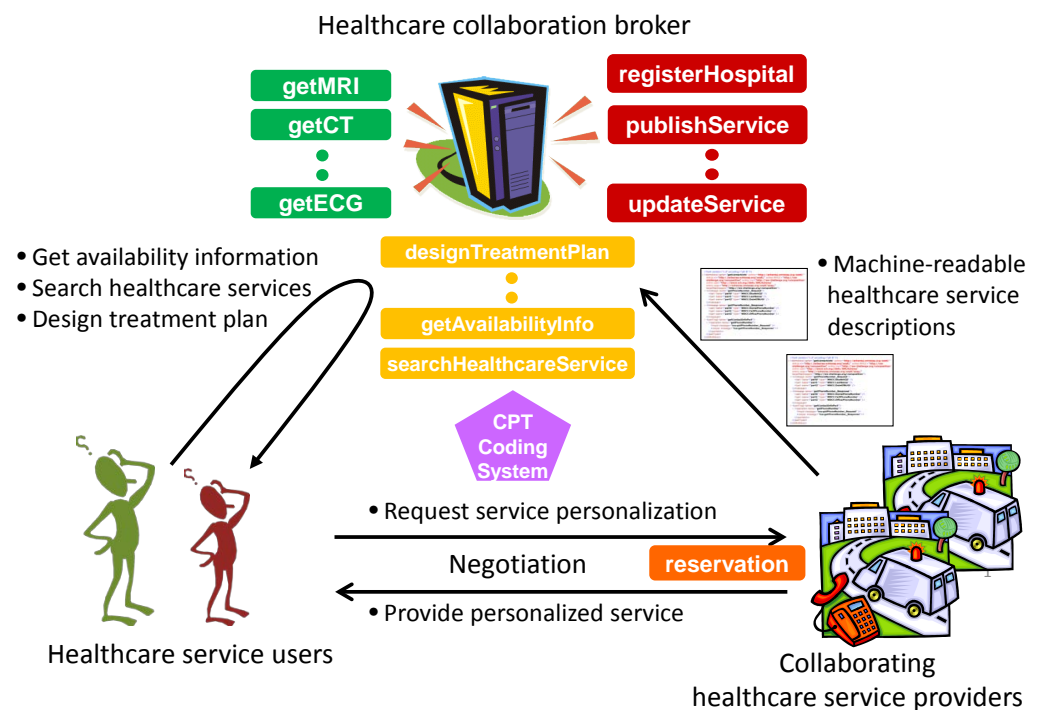

Figure 1. Collaborative healthcare service framework. 
care services, the broker needs to ascertain available services provided by the healthcare service providers that participate in the medical collaboration. The healthcare service providers share with the collaboration broker detailed information about their healthcare services. The information includes not only all the details of each healthcare service but also general hospital information, such as addresses or phone numbers. The detailed information about each healthcare service contains the CPT (Current Procedure Terminology) code for the healthcare service, availability, service lead time, doctor specializations, service fees, and etc. As shown in Figure 1, the CPT code plays a central role in the integration of the three major players in the proposed framework. When a healthcare service user searches a service, he or she will use the CPT code to precisely indicate which healthcare service he or she is looking for. When individual hospitals share their service information with the healthcare service broker, they will use the CPT code to describe the list of healthcare services that they can provide. In addition, the CPT code will be used for billing processes between hospitals and insurance companies.

Healthcare services are defined as activities for patients conducted by healthcare service providers, such as tests or operations. In order to share available resources for healthcare services seamlessly among collaborating hospitals, healthcare services need to be described precisely. A machine-readable language based on Extensible Markup Language (XML) [17] is recommended for the representation of healthcare services. A preliminary version of Healthcare Service Description Language (HSDL) is proposed in Figure 2. An HSDL description of a healthcare service includes service provider information, service fees, output information as a result of the healthcare service, and most importantly, the CPT code. Healthcare service providers describe their available services in HSDL and upload the descriptions to the collaboration broker's repository.

In order to standardize and automate the access to the healthcare service repository, the collaboration broker should provide subscription, publishing services for healthcare service providers and query services for healthcare service users. Those services are categorized into 1) managerial services and 2) query services. Managerial services consist of web services for subscription and un-subscription of healthcare service providers. Query services are provided for health care service users.

\subsection{Service Scenarios}

A medical collaboration broker, healthcare service users and healthcare service providers are three main players in the proposed framework. The proposed collaboration broker system provides web services for healthcare service providers, such as publishing, updating, deleting healthcare services. In addition, the broker system provides web services for healthcare service users, such as searching for a healthcare service or generating workflow that consists of multiple healthcare services. Searching for a healthcare service can be accomplished by invoking a web service. On the other hand, the web service for workflow generation arranges multiple healthcare services in a required sequence, which is pre-specified by medical doctors. While integrating multiple healthcare services, the web service for workflow generation utilizes single web service for healthcare service search multiple times.

Figure 3 illustrates a workflow generation process works using the UML sequence diagram [18]. Tradi-

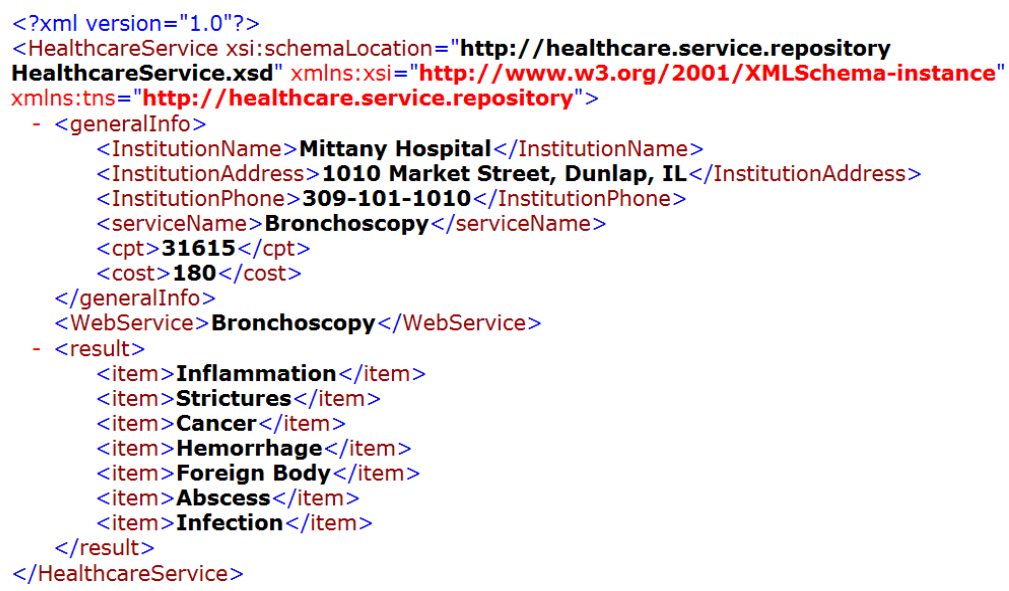

Figure 2. Healthcare service description language for bronchoscopy. 


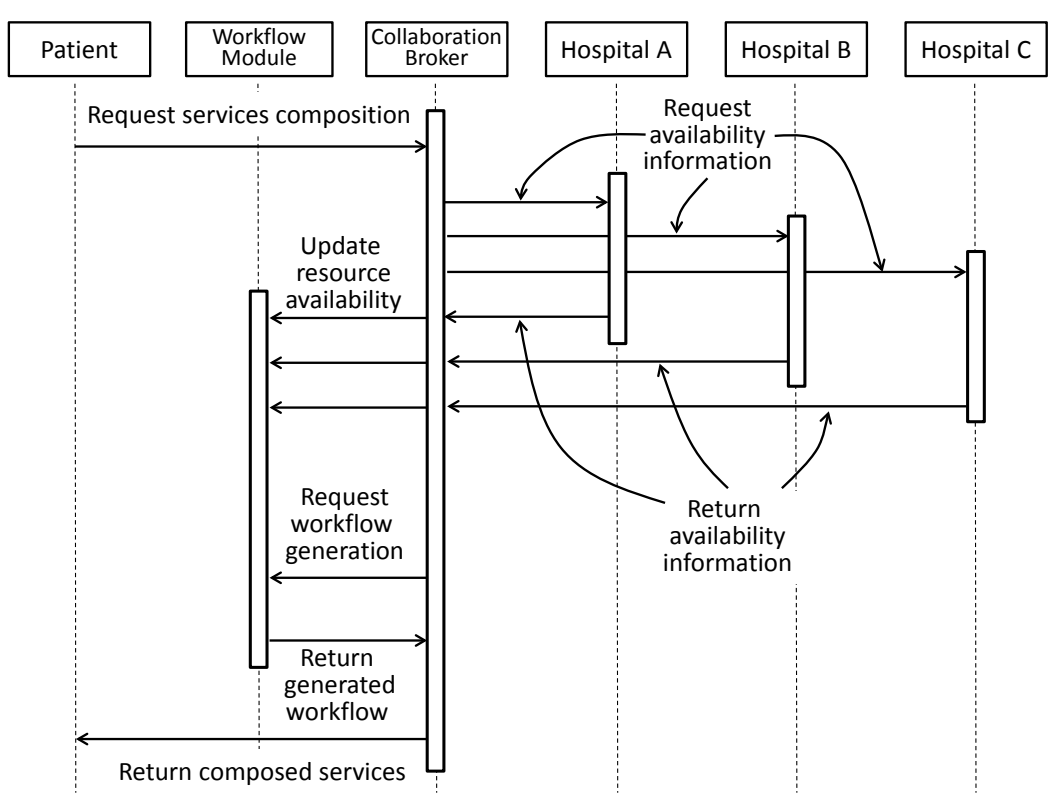

Figure 3. UML sequence diagram for collaborative healthcare service scenarios.

tionally, a patient who needs to receive multiple medical examinations would go to one hospital and undergo all four tests based on the resource availability of the hospital. In the proposed collaboration framework, however, the CPT code of the multiple medical examinations will be sent to hospitals participating in healthcare service collaboration via the proposed collaboration broker. Once the broker collects all availability information from the participating hospitals, the workflow generation module in the broker system will recommend the best healthcare service workflow based on the patient's priority, such as time, cost, or quality.

Figure 4 illustrates four different healthcare service workflows generated by the collaboration broker system for a patient who has symptoms of a liver cancer. The patient is advised to go through four tests for accurate diagnosis: Abdominal ultrasound, CT scan, MRI and liver biopsy. Abdominal ultrasound is performed first, followed by CT scan and MRI, and finally liver biopsy. Between CT scan and MRI, the doctor does not set any priority in sequence, thus either one can be conducted first. Next, liver biopsy is performed. Depending on the intermediate test results, the doctor will decide whether or not the rest of tests should be followed.

In the proposed collaborative healthcare service framework, the healthcare service coordinator requests a query to the collaboration broker with the CPT codes of four recommended medical tests and their sequence requirements. Figure 4 shows four different workflows based on four possible objectives. First, second, third, and fourth workflow have the best medical treatment plan in terms of time, cost, reputation and the number of hospitals, respectively. The resulting healthcare service workflow is generated in a Business Process Execution Language (BPEL) document [19].

\section{Simulation Study}

A simulation study is conducted to verify the performance of the proposed collaborative healthcare service framework compared to the current practice, which can be regarded as the non-collaborative healthcare service framework. This section describes the two healthcare service models, collaborative and non-collaborative service models, which are compared in this simulation study. Two performance measures are defined and used for the comparison of the two models. Finally, the simulation results and statistical analysis results are discussed in detail in this section.

\subsection{Collaborative and Non-Collaborative Healthcare Service Models}

Two healthcare service models, non-collaborative service model (NSM) and collaborative service model (CSM) are designed to represent the current practice and the proposed framework, respectively. The performance of the two models is compared in this simulation study. In NSM, patients have a primary healthcare service provider 


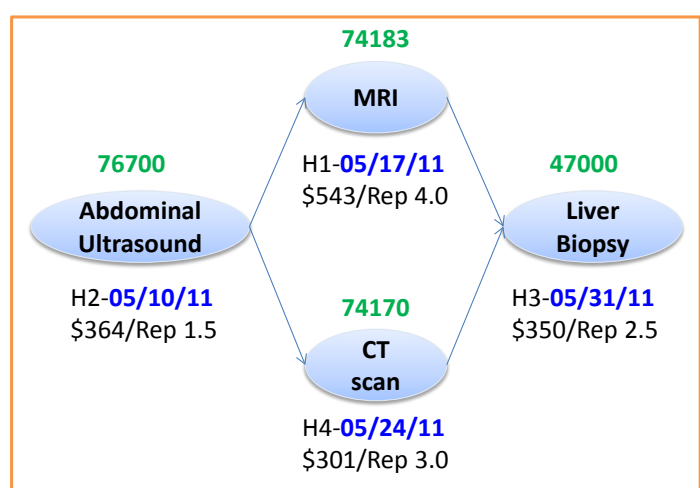

(a) Time

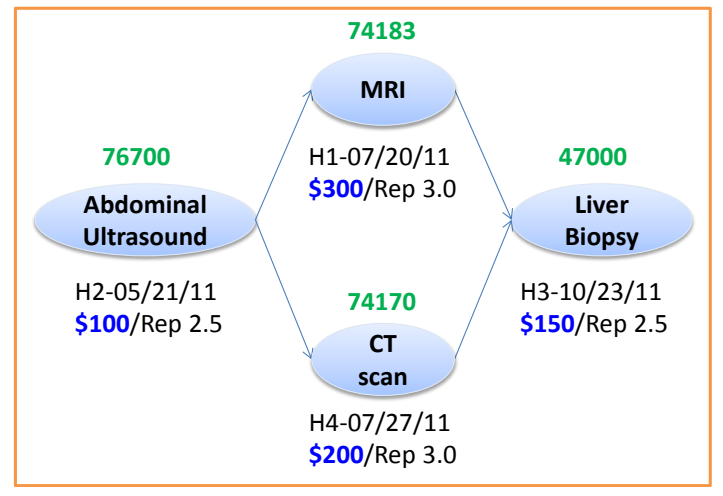

(c) Cost

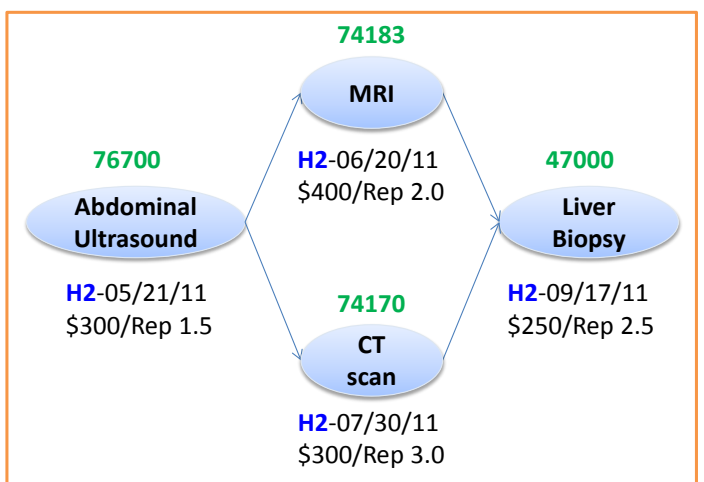

(b) Number of Hospital

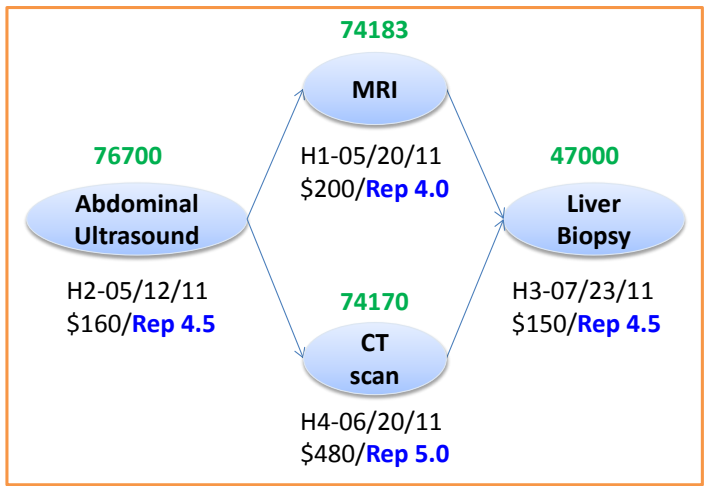

(d) Reputation

Figure 4. Liver cancer diagnosis processes in terms of four different objectives.

and receive all the treatments completed with the healthcare service provider. For example, if a patient needs a chest X-ray test and a sputum cytology test, the tests will be conducted by the service provider regardless of how early both tests are available with the service provider. On the other hand, in CSM, patients have a primary healthcare service provider, but can choose any healthcare service provider based on their needs, such as time or cost. For example, if a patient needs the two aforementioned tests at the earliest time, the patient can choose a service provider that has an earlier available schedule than other providers. During the service provider selection process, healthcare service coordinators, which are discussed in Section 3, will provide professional consulting services. These two service models are illustrated in Figure 5. In NSM, Hospital A is the primary service provider for the patients in dark (or green) color, which implies that they receive all healthcare services in Hospital A without any exception. In the same way, the patients in light (or gray) color get all services from Hospital B, which is their primary service provider. On the other hand, in CSM, the patients in Hospital A and B consist of patients in dark (or green) color and patients in light (or gray) color, which imply that they choose any healthcare service provider.

\subsection{Experimental Design}

In the simulation study we have considered four hospitals, denoted by $\mathrm{H} 1$ through $\mathrm{H} 4$, and three different treatments, denoted by T1 through T3. Four different patient types (PT), denoted by PT1 through PT4, are defined based on the series of treatments that a patient needs to receive. The treatment sequence for Patient type 1 (PT1) is $\mathrm{T} 1 \rightarrow \mathrm{T} 2$; that of PT2 is $\mathrm{T} 2 \rightarrow \mathrm{T} 3$; that of PT3 is $\mathrm{T} 1 \rightarrow \mathrm{T} 3$; and that of PT4 is $\mathrm{T} 1 \rightarrow \mathrm{T} 2 \rightarrow \mathrm{T} 3$. The sequence of treatments is maintained in the simulation model by allowing the succeeding treatment to start only after completion of the preceding treatment. Inter-arrival time of patients is considered to be exponentially distributed and treatment times are constant. In order to consider various scenarios at individual hospitals, ten scenarios of different inter-arrival and treatment times are considered as shown in Table 2. Both models are set to run at the nine experimental scenarios for thirty replications per scenario. Inter-arrival times and treatment times are con- 


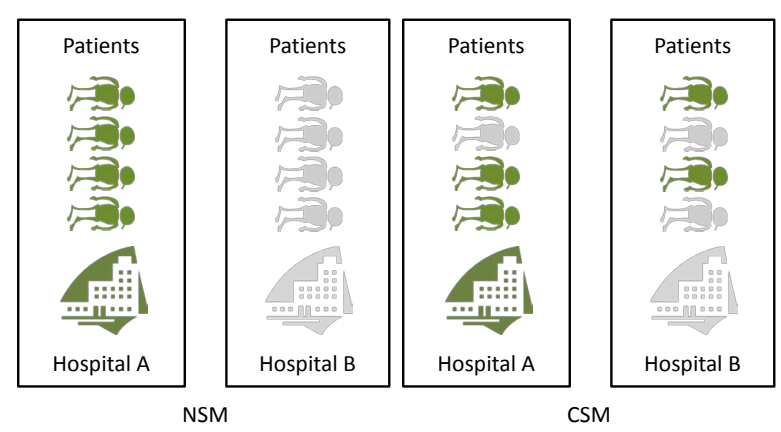

Figure 5. Collaborative and non-collaborative healthcare service models.

\begin{tabular}{ccccc}
\multicolumn{5}{l}{ Table 2. Experimental design. } \\
\hline No. & Inter-arrival Time (min) & Treatment1 (min) & Treatment 2 (min) & Treatment 3 (min) \\
\hline 1 & 55 & 20 & 25 & 20 \\
2 & 60 & 20 & 25 & 20 \\
3 & 60 & 20 & 25 & 30 \\
4 & 75 & 25 & 25 & 25 \\
5 & 75 & 20 & 25 & 30 \\
6 & 85 & 20 & 25 & 20 \\
7 & 90 & 20 & 25 & 30 \\
8 & 90 & 25 & 25 & 25 \\
9 & 95 & 20 & 25 & 25 \\
\hline
\end{tabular}

sidered to be exponentially distributed. The detailed experimental design is given in Table 2. The Pro Model simulation software is used to develop the simulation model for the collaborative healthcare service model (CSM) and the non-collaborative healthcare service model (NSM).

To compare the performance of the CSM and the NSM, two performance measures, resource utilization and treatment lead time, are used. The definition of the two performance measures are as follows.

1) Resource Utilization (RU): Percentage of resource usage in terms of time.

$$
\text { RU = Occupied Time/Available Time }
$$

2) Treatment Lead Time (TLT): Total time taken to complete all the treatments for all patients including waiting time.

$$
\text { TLT }=\text { Treatment Time }+ \text { Waiting Time }
$$

\subsection{Experimental Results}

A statistical analysis is conducted to verify the advantages of the proposed collaborative service model (CSM) over the current non-collaborative service model (NSM). Two paired t-tests are performed to identify whether or not the collaborative model results in increased resource utilization (RU) and decreased treatment lead time (TLT) against the non-collaborative model. The null and alternative hypotheses for treatment lead time are $\mathrm{H}_{0}$ : $\mu_{C S M}^{T L T}=\mu_{N S M}^{T L T}$ and $H_{1}: \mu_{C S M}^{T L T} \neq \mu_{N S M}^{T L T}$, where $\mu_{C S M}^{T L T}$ and $\mu_{N S M}^{T L T}$ are the average treatment lead time in the collaborative service model (CSM)and the non-collaborative service model (NSM), respectively. On the other hand, the null and alternative hypotheses for resource utilization are $\mathrm{H}_{0}: \mu_{C S M}^{R U}=\mu_{N S M}^{R U}$ and $\mathrm{H}_{1}: \mu_{C S M}^{R U} \neq \mu_{N S M}^{R U}$, where $\mu_{C S M}^{R U}$ and $\mu_{N S M}^{R U}$ are the average resource utilization in the CSM and the NSM, respectively.

Table 3 summarizes the results of the experiments conducted based on the experimental design described in the previous section, where the CSM outperforms the NSM with statistical significance in both performance measures. Paired t-tests are conducted. The p-values for TLT and RU are 0.001 and 0.017 , respectively. Therefore, we conclude that the two models yield different results. In summary, the CSM has less treatment lead time 
Table 3. Experimental results.

\begin{tabular}{ccccccc}
\hline \multirow{2}{*}{ No. } & \multicolumn{3}{c}{ Resource Utilization (\%) } & \multicolumn{3}{c}{ Treatment Lead Time (minute) } \\
\cline { 2 - 7 } & Non-collaborative & Collaborative & Difference (\%) & Non-collaborative & Collaborative & Difference (\%) \\
\hline 1 & 87.0 & 88.7 & 1.9 & 200 & 132 & 33.8 \\
2 & 81.2 & 83.0 & 2.1 & 145 & 100 & 30.8 \\
3 & 91.3 & 92.9 & 1.7 & 309 & 206 & 33.4 \\
4 & 74.9 & 75.5 & 0.8 & 139 & 101 & 27.4 \\
5 & 79.8 & 80.5 & 0.8 & 174 & 117 & 32.6 \\
6 & 58.1 & 58.2 & 0.2 & 143 & 70 & 51.4 \\
7 & 63.3 & 63.4 & 0.2 & 104 & 82 & 20.9 \\
8 & 63.4 & 63.5 & 0.1 & 104 & 85 & \\
9
\end{tabular}

(TLT) and more resource utilization (RU) than does the NSM.

On average, $29.4 \%$ reduction in treatment lead time (TLT) is expected by utilizing the collaborative service model (CSM) over the non-collaborative service model (NSM). Particularly, Scenarios 1 through 6 have much higher reduction in TLT than the other scenarios. This finding shows that hospitals with higher utilization in NSM can expect higher reduction in TLT than hospitals with lower utilization. On the other hand, in terms of resource utilization on average $0.9 \%$ increase in resource utilization is expected. In particular, hospitals with higher resource utilization, shown in Scenarios 1 through 5, have relatively higher increase in resource utilization than hospitals with lower resource utilization.

\section{Conclusions}

This paper proposes a framework for collaborative healthcare services in order to facilitate collaborative services among hospitals to provide reduced treatment lead time for patients and increased utilization of medical resources of hospitals. The service scenarios are discussed in detail, which take into account four different considerations of patients to show how healthcare services can be provided based on the proposed framework. In order to verify the advantages of the proposed framework, a simulation study is conducted. The current practice of the non-collaborative service model (NSM) and the proposed framework of the collaborative service model (CSM) are compared using paired t-test. The simulation experiment results show that the proposed framework can contribute not only to the increase of hospital resource utilization, but also to the reduction of treatment lead time.

The Current Procedure Terminology (CPT) code plays a key role in the framework. The framework provides various services, such as searching medical services, integrating them and generating workflows, using the coding system. The unique identification capability of the CPT code enables accurate search for services provided by healthcare service providers. Although the proposed architecture adopts the Current Procedure Terminology (CPT) code to identify specific healthcare services, the architecture of the proposed framework is not dependent on the CPT coding system and can easily adopt alternative coding systems. The coding system has kept improving to reflect the practical needs. As the coding system improves, the proposed framework is expected to provide more accurate services.

As a future research topic, a mathematical model should be designed to generate the optimal treatment plan according to the objective of the service users. The mathematical model should be able to incorporate multiple objectives because healthcare-related decisions are normally made considering multiple aspects. These mathematical models should be integrated with the real-time availability information provided by healthcare service providers via the proposed healthcare collaboration broker. Considering the complexity of the treatment plan generation, a heuristic algorithm should also be developed to provide real-time collaborative healthcare services. If the treatment plan is urgently required, the heuristic algorithm can be utilized. Otherwise, the mathematical model can be used. The mathematical model can be also used as a benchmark tool for the performance of the heuristics algorithm. 


\section{References}

[1] Nolan, R.L. and Kotha, S. (2005) Boeing 787: Dreamliner. Harvard Business School Case Study, Compilation 305101.

[2] Burgess, J.F., Carey, K. and Young, G.J. (2005) The Effect of Network Arrangements on Hospital Pricing Behavior. Journal of Health Economics, 24, 391-405. http://dx.doi.org/10.1016/j.jhealeco.2004.10.002

[3] Getzenberg, J., Cohen, S., Herd, J., Sayer, J. and Vandebroek, K. (2008) The Chicago Health Care Access Puzzle: Fitting the Pieces Together. Chicago Department of Public Health, Office of Policy \& Planning, Chicago.

[4] Wachter, R.M. (2006) The "Dis-Location” of U.S. Medicine-The Implications of Medical Outsourcing. The New England Journal of Medicine, 354, 661-665. http://dx.doi.org/10.1056/NEJMp058258

[5] Lai, I.K.W., Tam, S.K.T. and Chan, M.F.S. (2012) Knowledge Cloud System for Network Collaboration: A Case Study in Medical Service Industry in China. Expert Systems with Applications, 39, 12205-12212. http://dx.doi.org/10.1016/j.eswa.2012.04.057

[6] Martinez, P., Guillama, N.J. and Heath, C. (2009) Medical Equipment Allocation and Utilization System and Method. The Quantum Group, Inc.

[7] Steins, K., Persson, F. and Holmer, M. (2010) Increasing Utilization in a Hospital Operating Department Using Simulation Modeling. Simulation, 86, 463-480 http://dx.doi.org/10.1177/0037549709359355

[8] Gunter, T.D. and Terry, N.P. (2005) The Emergence of National Electronic Health Record Architectures in the United States and Australia: Models, Costs, and Questions. Journal of Medical Internet Research, 7, e3. http://dx.doi.org/10.2196/jmir.7.1.e3

[9] Shaver, D. (2010) The HL7 Evolution. Last accessed on June 27, 2013. http://www.corepointhealth.com/whitepapers/evolution-hl7

[10] Kimura, M., Ohe, K., Yoshihara, H., Ando, Y., Kawamata, F., Tsuchiya, F., Furukawa, H., Horiguchi, S., Sakusabe, T., Tani, S. and Akiyama, M. (1998) MERIT-9: A Patient Information Exchange Guideline Using MML, HL7 and DICOM. International Journal of Medical Informatics, 51, 59-68. http://dx.doi.org/10.1016/S1386-5056(98)00090-2

[11] Hori, M., Ohashi, M. and Suzuki, S. (2005) Citizen-Centric Approach and Healthcare Management Based on the XML Web Services. The Electronic Journal Information Systems Evaluation, 8, 179-186.

[12] Hori, M. and Ohashi, M. (2005) Applying XML Web Services into Health Care Management. Proceedings of International Conference on System Sciences, Hawaii, 3-6 January 2005.

[13] Dunphy, G., Campos, H., Kaufman, S., Kelcey, P., Moukhnitski, S. and Peterson, D. (2009) Pro BizTalk 2009. Apress, New York. http://dx.doi.org/10.1007/978-1-4302-1982-8

[14] Lee, Y., Patel, C. and Chun, S. A. (2004) Towards Intelligent Web Services for Automating Medical Service Composition. Proceedings of IEEE International Conference on Web Services, San Diego, 6-9 July 2004, 384-391.

[15] Alexander, S., Conner, T. and Slaughter, T. (2003) Overview of Inpatient Coding. American Journal of Health-System Pharmacy, 60, S11-S14.

[16] Erl, T. (2004) Service-Oriented Architecture: A Field Guide to Integrating XML and Web Services. Prentice Hall, Upper Saddle River.

[17] Bray, T., Paoli, J., Sperberg-McQueen, C.M., Maler, E. and Yergeau, F. (2008) Extensible Markup Language (XML) 1.0. Last accessed on June 27, 2013. http://www.w3.org/TR/REC-xml/

[18] Rumbaugh, J., Jacobson, I. and Booch, G. (2004) The Unified Modeling Language Reference Manual. Addison-Wesley Professional, Boston.

[19] Margolis, B. (2007) SOA for the Business Developer: Concepts, BPEL, and SCA. Mc Press, Lewisville. 
Scientific Research Publishing (SCIRP) is one of the largest Open Access journal publishers. It is currently publishing more than 200 open access, online, peer-reviewed journals covering a wide range of academic disciplines. SCIRP serves the worldwide academic communities and contributes to the progress and application of science with its publication.

Other selected journals from SCIRP are listed as below. Submit your manuscript to us via either submit@scirp.org or Online Submission Portal.
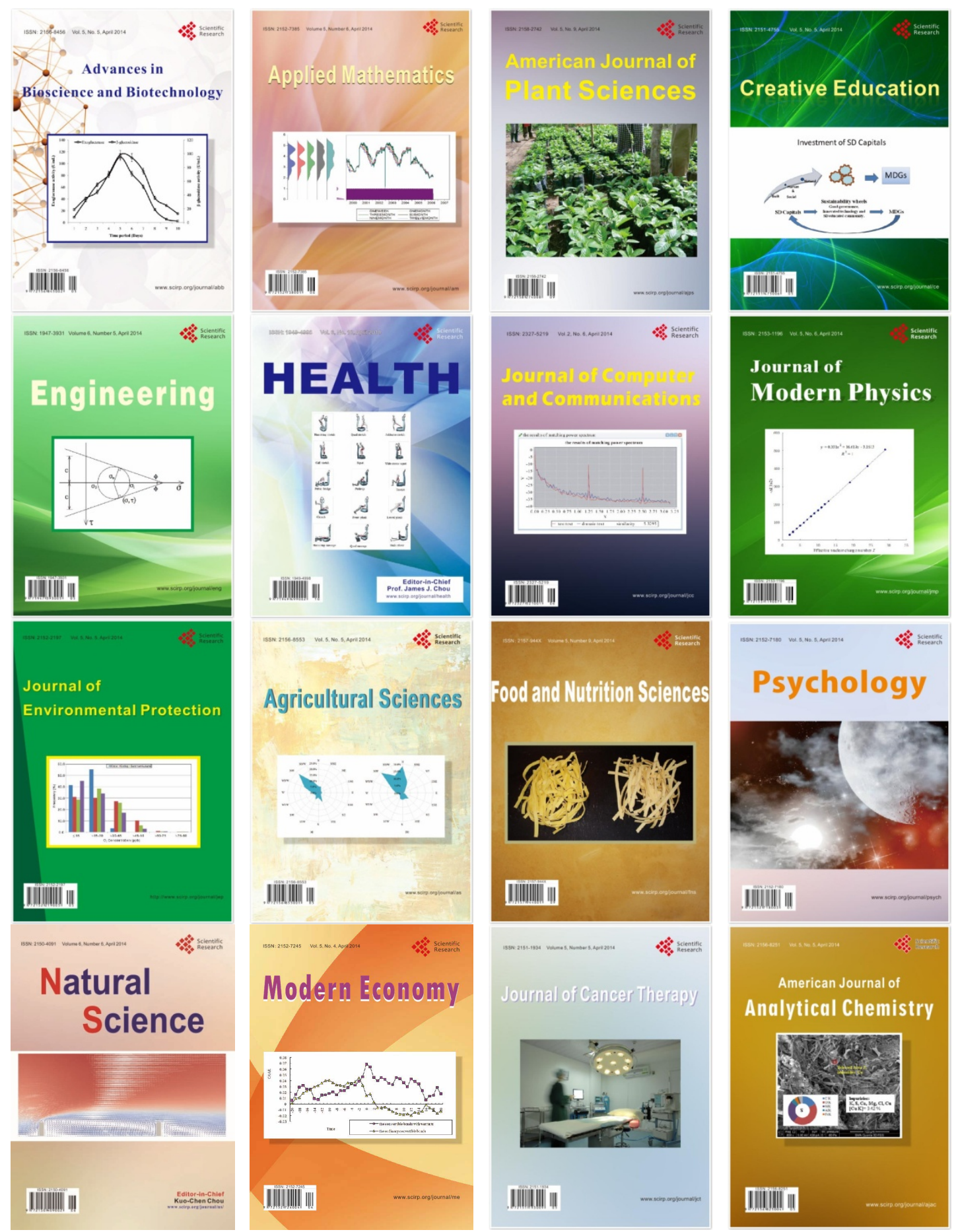